\title{
Asymetrical limbs arterial pressures: a new marker of atherosclerosis
}

\author{
Victor Aboyans \\ Hypertension Research (2013) 36, 394-395; doi:10.1038/hr.2012.228; published online 24 January 2013
}

$\mathrm{T}$ he development of atherosclerosis is a long-standing process that expands unequally throughout the large vessels within the arterial network. For reasons that are not yet fully clarified, the distribution of this disease is heterogeneous. Even in the bilateral arteries of a same person, the distribution of atherosclerotic lesions is often asymmetric. Patients with carotid artery disease present mostly an unbalanced pattern, with a unilateral significant stenosis in almost $90 \%$ of cases. Accordingly, the distribution of plaques and significant stenosis in intracranial arteries is heterogeneous. Similarly, the majority of patients ( $>80 \%$ of cases) with renal artery disease due to atherosclerosis present with unilateral stenosis.

Although the identification of atherosclerosis in the aforementioned arteries requires sophisticated imaging techniques, the presence of hemodynamically significant stenotic lesions in the arteries of the limbs can be identified by the arterial pressure drop. Similar to other localizations of atherosclerosis, lower extremities peripheral artery disease (PAD) is often asymmetrically distributed. In population studies using the measurement of the ankle-brachial index $(\mathrm{ABI})$ to diagnose PAD, one-half of the cases are unilateral, equally distributed between both sides.

In the arms, the most frequent localization of atherosclerosis is in the subclavian arteries, including the innominate artery. Here again, the vast majority of cases are unilateral, so that a significant inter-arm blood pressure difference (IABPD) occurs. There are only two studies that have assessed the ability

$\mathrm{V}$ Aboyans is at Department of Cardiology, Dupuytren University Hospital, Limoges, France

E-mail: vaboyans@live.fr of the IABPD measurement to predict angiographic $>50 \%$ subclavian stenosis, ${ }^{1,2}$ both performed on patients undergoing coronary angiography. In a series of 492 patients, English et al. reported a sensitivity of 65 and $35 \%$ to predict $>50 \%$ subclavian stenosis for an IABPD of $>10$ and $>20 \mathrm{~mm} \mathrm{Hg}$, respectively, with specificities at 85 and $94 \%$. In another series of 59 patients undergoing coronary bypass surgery, the sensitivity and specificity of an IABPD $>15 \mathrm{~mm} \mathrm{Hg}$ were both at $100 \%$. The population distribution of an IABPD has been studied by Shadman et al. ${ }^{3}$ who reported the 5th and 95th percentiles of two community-dwelling cohorts at -9 and $+9 \mathrm{~mm} \mathrm{Hg}$, respectively. In another large study in Japan, ${ }^{4}$ the average right minus left arm systolic blood pressure was $-0.6 \pm$ $6.6 \mathrm{~mm} \mathrm{Hg}$, which gives a $95 \%$ confidence interval of -13.8 to $+12.6 \mathrm{~mm} \mathrm{Hg}$. In a systematic review of four studies of unselected individuals with simultaneous blood pressure measurement in both arms, Clark et al. ${ }^{5}$ reported the prevalence of an IABPD of $\geqslant 10$ and $\geqslant 20 \mathrm{~mm} \mathrm{Hg}$ at $19.6 \%$ and $4.2 \%$, respectively. In general populations, the prevalence of an IABPD of $>15 \mathrm{~mm} \mathrm{Hg}$ ranges from 1.9 to $4.6 \% .^{3,6}$

Similar to lower extremities PAD in the lower extremities, the presence of subclavian stenosis as revealed by an elevated IABPD is prognostic. In the Multi-Ethnic Study of Atherosclerosis, the presence of an IABPD of $>15 \mathrm{~mm} \mathrm{Hg}$ was associated with an increased risk of PAD, an increased coronary calcium score and an increase in carotid intima-media thickness. ${ }^{6}$ In a meta-analysis of community-dwelling cohorts, an IABPD of $\geqslant 15 \mathrm{~mm} \mathrm{Hg}$ was associated with a $55 \%$ increased risk of death and a $68 \%$ increased risk of cardiovascular death. ${ }^{7}$ Therefore, the estimation of the IABPD can be considered as a marker for cardiovascular disease and prognosis.

In this issue, Okada et al. ${ }^{8}$ not only confirmed the usefulness of determining the IABPD in diabetic patients, but also found that similar findings between both legs may also be of interest. The authors assessed urinary albumin excretion and brachialankle pulse wave velocity, two well-known markers for cardiovascular prognosis, and found an association between an IABPD of $\geqslant 10 \mathrm{~mm} \mathrm{Hg}$ and/or an inter-leg BPD of $\geqslant 15 \mathrm{~mm} \mathrm{Hg}$ with the two markers in the patients they studied. The latter difference is by far higher than the one expected in healthy subjects. Importantly, the average ABI of these patients was at 1.11 , so that the majority had normal ABIs. This suggests that a number of patients without PAD may have an important inter-leg BPD and that this condition would be associated with the other markers of cardiovascular risk, although this specific issue has regrettably not addressed in this paper. The potential incremental value of inter-leg BPD to that provided by the ABI remains to be clarified. Also, as highlighted by the authors, their study is cross-sectional, so that the hypothesis engendered above requires confirmation through longitudinal studies.

The reason why an inter-leg BPD occurs is not only related to the asymmetrical development of atherosclerosis, which leads to lower pressure in the most severely affected leg, but is also related to the possibility of the asymmetrical development of medial calcinosis (MC), which leads to a pressure overestimation in the most severely affected leg. A diabetic patient can present both diseases together, as more than half of patients with MC present with simultaneous 
arterial stenosis, ${ }^{9}$ so that predominantly stiffened arteries may lead to a pressure overestimation in one leg, whereas predominantly stenotic lesions in the other leg may lead to a pressure drop in the contralateral leg. According to its distribution presented in their paper, Okada et al. ${ }^{8}$ found an inter-leg $\mathrm{BPD}$ of $\geqslant 15 \mathrm{~mm} \mathrm{Hg}$ in $>15 \%$ of cases. As diabetes is the most powerful predictor of MC in leg arteries, ${ }^{9}$ it is unclear whether similar magnitudes of inter-leg BPD may be present in non-diabetic individuals. Similarly, the association of this condition with other markers of atherosclerosis in non-diabetic individuals is not known.

If these findings are assessed in future studies, special attention should be given to the methods used. The current paper presents good methodology, with concomitant measurement of systolic pressures of the four limbs. Sequential measurement is associated with higher intra- and inter-observer variability and higher probability of finding increased inter-limb BPD. In contrast, the use of an oscillometric device precludes the determination of all ankle artery pressures, as these devices determine only the highest pressure among the three ankle arteries.
Whether pressure disparities among the arteries of the same ankle could also provide additional information is plausible but requires specific studies.

The paper by Okada et al. ${ }^{8}$ reveals a new path in the search for easily accessible markers for atherosclerosis and cardiovascular prognosis, and from this standpoint they should be commended. Further studies, especially in general population, are necessary to determine whether, along with the inter-arm BPD, the inter-leg BPD could be on the short list for cardiovascular markers that can identify easily and at low cost those at increased risk of cardiovascular events.

\section{CONFLICT OF INTEREST}

The author declares no conflict of interest.

1 English JL, Carell ES, Guidera SA, Tripp HF. Angiographic prevalence and clinical predictors of left subclavian stenosis in patients undergoing diagnostic cardiac catheterization. Cathet Cardiovasc Intervent 2001; 54: 8-11.

2 Osborn LA, Vernon SM, Reynolds B, Timm C, Allen K. Screening for subclavian stenosis in patients who are candidates for coronary bypass surgery. Cathet Cardiovasc Intervent 2002; 56: 162-165.

3 Shadman R, Criqui MH, Bundens WP, Fronek A, Denenberg JO, Gamst AC, McDermott MM. Subclavian stenosis: the prevalence, risk factors and association with other cardiovascular diseases. J AmColl Cardiol 2004; 44: 618-623.

4 Kimura A, Hashimoto J, Watabe D, Takahashi H, Ohkubo T, Kikuya M, Imai Y. Patient characteristics and factors associated with inter-arm difference of blood pressure measurements in a general population in Ohasama, Japan. J Hypertens 2004; 22: 2277-2283.

5 Clark CE, Campbell JL, Evans PH, Millward A. Prevalence and clinical implications of the inter-arm blood pressure difference: a systematic review. J Hum Hypertens 2006; 20: 923-931.

6 Aboyans V, Kamineni A, Allison MA, McDermott MM, Crouse JR, Ni H, Szklo M, Criqui MH. The epidemiology of subclavian stenosis and its association with markers of subclinical atherosclerosis: The MultiEthnic Study of Atherosclerosis (MESA). Atherosclerosis 2010; 211: 266-270.

7 Clark CE, Taylor RS, Shore AC, Ukoumunne OC, Campbell JL. Association of a difference in systolic blood pressure between arms with vascular disease and mortality: a systematic review and meta-analysis. Lancet 2012; 379: 905-914.

8 Okada H, Fukui M, Tanaka M, Matsumoto S, Mineoka Y, Nakanishi N, Asano M, Yamazaki M, Hasegawa G, Nakamura N. A difference in systolic blood pressure between arms and between lower limbs is a novel risk marker for diabetic nephropathy in patients with Type 2 diabetes. Hypertens Res 2013; 36: 403-407.

9 Aboyans V, Ho E, Denenberg JO, Ho LA, Natarajan L, Criqui $\mathrm{MH}$. The association between elevated ankle systolic pressures and peripheral occlusive arterial disease in diabetic and nondiabetic subjects. J Vasc Surg 2008; 48: 1197-1203. 